\title{
FLEBOTOMÍNEOS DA FLORESTA DE TERRA FIRME DA AMAZÔNIA PERUANA (DIPTERA: PSYCHODIDAE).
}

\author{
Marcos CABANILLAS ${ }^{1}$, Janeth BRAGA ${ }^{2}$, Máximo VIENA ${ }^{3}$
}

RESUMO - Um total de 1030 flebotomíneos, pertencentes a 14 espécies, foram coletados com armadilha tipo Shannon e isca humana, nos meses de Julho e Agosto de 1991, nos horários das 18:00 h. às 21:00 h, nas localidades de San Juan, San Pedro e 25 de Diciembre localizadas na Amazônia Peruana. As fêmeas foram mais abundantes do que os machos (96,98\% e 3,02\% respectivamente). Os subgêneros Psychodopygus (49,27\% e 7 spp. coletadas) e Nyssomyia ( $48,79 \%$ e $4 \mathrm{spp}$, coletadas) comportaram o maior número de espécies e indivíduos. As espécies Lutzomyia richardwardi $(48,4 \%)$ e $L$. carrerai $(29,2 \%)$ foram as mais abundantes. Na localidade de San Juan foi coletado o maior número de indivíduos $(55,2 \%)$, porém o maior número de espécies foi obtido na localidade de 25 de Diciembre. Quanto a estratégia de coleta, o mesmo número de espécies (12 spp.) foram obtidos com ambas as armadilhas, porém um maior número de individuos foi capturado com a técnica de isca humana.

Palavras-chave: flebotomineos, armadilha Shannon, isca humana, Amazônia Peruana.

\section{Phlebotomine Sandflies from Non-flooded Forest in the Peruvian Amazon (Diptera: Psychodidae).}

\begin{abstract}
A total of 1,030 phlebotomines sandflies, belonging to 14 species was collected with a trap type Shannon and human bait, during the season of less rainfall, from 18:00h. to 21:00 h. in the localities of San Juan, San Pedro and 25 de Diciembre located in the Peruvian Amazon. Females outnumbered males ( $96.98 \%$ and $3.02 \%$ respectively). The subgenus Psychodopygus (49. 27\% and 7 species collected) and Nyssomyia (48.79\% and 4 species collected) had the highest numbers of species and specimens, Lutzomyia (Nyssomyia) richardwardi (48.4\%) and L.(Psychodopygus) carrerai $(29.2 \%)$ presented the highest numbers of specimens. The highest number of specimens (55.2\%) was collected in San Juan, however, the highest number of species was collected in 25 de Diciembre. The same numbers of species ( 12 species) was collected by both collection strategies, however, the highest number of specimens was collected with human bait technique.
\end{abstract}

Key-words: phlebotomine, Shannon trap, human bait, Peruvian Amazon.

\section{INTRODUÇÃO}

As leishmanioses nas Américas representam um problema de saúde pública para as populações humanas que moram em áreas endêmicas e de transmissão ativa, assim como também para o pessoal que trabalha em projetos agro - florestais, de pesquisa e construção de estradas nessas áreas (Lainson, 1988).

No Peru, trabalhos sobre a biologia, comportamento e sistemática de flebotomíneos, limitaram-se principalmente às áreas das encostas andinas e de transição entre Serra e Floresta (Herrer, 1977, 1982; Young et al., 1985; Peréz et al., 1987; Cáceres et al., 1995; Fernandez et al., 1998). Na Amazônia Peruana, o caráter silvestre das diversas formas de leishmaniose tegumentar, sem conhecimento claro dos principais transmissores, e com pesquisas que visam apenas aspectos sistemáticos e de distribuição geográfica de algumas 
espécies(Llanos, 1964, 1973, 1986; Llanos et al., 1975; Fraiha et al., 1980, Fernandez et al., 1998 ), são de pouca ajuda no planejamento de programas direcionadas ao vetor e diminuem a efetividade das ações de controle sobre esta importante endemia.

Nos últimos anos, a expansão da fronteira agricola, a extração agressiva de madeira e a colonização de novas áreas para o estabelecimento de grupos humanos, sem levar em consideração critérios técnicos e sanitários, tem ocasionado um incremento no número de casos de leishmaniose devido principalmente a Leishmania (Viannia) braziliensis, em vários locais da Amazônia Peruana (Braga, 1987). Justifica-se assim a necessidade de estudar aspectos do comportamento (antropofilia) e distribuição das espécies de flebotomíneos presentes em 3 localidades próximas onde casos de leishmanioses foram reportados.

\section{MATERIAL E MÉTODOS Área de Estudo}

Foram consideradas três $(03)^{\circ}$ localidades (San Juan, San Pedro e 25 de Diciembre) localizadas no município de Yurimaguas, Provincia do Alto Amazonas, do Estado de Loreto, Peru. O município de Yurimaguas localiza-se entre as coordenadas geográficas, $76^{\circ} 20^{\prime}$ e $77^{\circ} 03^{\prime}$ 'W e $2,4^{\circ} 10^{\prime}$ e $6,3^{\circ} 28^{\prime} \mathrm{N}$, a uma altitude aproximada de $180 \mathrm{~m}$ a $\mathrm{n} \mathrm{m}$. Ecologicamente classifica-se como Floresta Úmida Tropical e ecozoogeograficamente como Selva Baixa
(Asesoria Nacional de Planificación, 1959; ONERN, 1976; Villarejo, 1979). A temperatura média anual é de 26,4 ${ }^{\circ} \mathrm{C}$, a média mínima de $21,6{ }^{\circ} \mathrm{C}$ e a média máxima de $31,3^{\circ}$ C. A precipitação anual média é de 2.125 $\mathrm{mm}$ e a umidade relativa média de $84 \%$. Os ventos dominantes são do $\mathrm{NE}$ com uma velocidade média de $5,5 \mathrm{~km} /$ h.(ONERN, 1976).

As três localidades encontram-se ao longo das margens do igarapé Zapote, o qual é um afluente do rio Huallaga. As áreas de coleta estavam localizadas dentro da floresta, de 250 a $500 \mathrm{~m}$ de distância das localidades. Características como maior quantidade de corpos de água dentro da floresta, diferenciam a paisagem ecológica entre uma localidade e outra.

\section{Metodologia}

As coletas foram realizadas em áreas de floresta primária, próximas a cada vila, na estação de menor precipitação pluviométrica (Julho e Agosto de 1991), com armadilhas de Shannon e isca humana em horários crepusculares - noturnos (18:00h. às 21:00h.)

As coletas com armadilha tipo Shannon foram realizadas segundo a técnica descrita por Perez et al. (1987), enquanto que as coletas com isca humana seguiram a técnica descrita por Feliciangeli (1987). Ambas as técnicas de coletas foram realizadas simultaneamente, a uma distância aproximada de 50 metros entre uma e outra.

Em ambas as técnicas de coleta utilizaram-se lanternas para a captura 
dos flebotomíneos, porém procuravase mantê-las ligado o mínimo tempo possível. Um período de 20 a 30 segundos na coleta com Isca humana, e na armadilha tipo Shannon por um periodo de 1 a 2 minutos, segundo a quantidade de flebotomíneos presentes. Salientamos que, nas coletas com armadilhas Shannon, os periodos de iluminação intercalavamse com períodos de escuridão, que durava $5 \mathrm{~min}$. aproximadamente.

No dia seguinte à coleta do material entomológico, os flebotomíneos eram triados e guardados a seco (Maroli et al., 1995) para posterior montagem e identificação. 'Para confirmar a identificação dos espécimes coletados foram utilizadas a literatura de Young (1979), as descrições e redescrições do material pesquisado.

Para análise dos nossos dados utilizamos o coeficiente de similaridade de Jaccard (Ludwig \& Reynolds, 1988) com a finalidade de medir o grau de associação de espécies de flebotomíneos entre as localidades trabalhadas.

\section{RESULTADOS}

Foi coletado um total de 1030 flebotomineos agrupados em 14 espécies, e com uma relação de fêmeas para machos de aproximadamente 32:1. Indicamos que as nossas análises estarão direcionadas principalmente para as fêmeas, devido a sua abundância e importância epidemiológica na transmissão das leishmanioses.

Sete espécies e $49,27 \%$ do total de individuos coletados foram do subgênero Psychodopygus, porém foram coletadas apenas 4 espécies de Nyssomyia, seguinte subgênero em ordem de abundância, com $48,79 \%$ do total de flebotomíneos coletados nas 3 localidades (Tab. 1).

\section{Lutzomyia}

(Nyssomyia)

richardwardi foi à espécie mais abundante em nossas coletas $(48,4 \%)$, seguida das espécies de Psychodopygus: L. carrerai $(29,2 \%)$, L. hirsuta $(7,7 \%)$, L. amazonensis $(6,7 \%)$, L. davisi $(3,4 \%)$ e outros $(4,6 \%)$. Embora $L$. richardwardi tenha sido a espécie mais abundante, dominou a fauna de flebotomineos apenas em 2 das 3 localidades que fizeram parte do presente trabalho (Tab. 1).

Quanto as localidades nas quais foram realizadas as coletas de flebotomineos, em San Juan coletaramse $55,2 \%(12 \mathrm{M}$ e $557 \mathrm{~F})$, em 25 de Diciembre $22,5 \%$ (13 M e $223 \mathrm{~F}$ ) e em San Pedro $22,3 \%$ (6 M e $220 \mathrm{~F}$ ) do total de indivíduos coletados (Tab. 1).

Analisando os dados por localidade, encontramos que em San Juan o subgênero Psychodopygus foi o mais abundante ao nivel de espécies ( $85 \%$ ), porém o subgênero Nyssomyia foi mais abundante ao nivel de indivíduos $(58,91 \%)$ com uma única espécie, L. richardwardi. Adicionamos que espécies de outros subgêneros não foram coletadas com as técnicas em questão (Tab. 1).

Na localidade de San Pedro os subgêneros Psychodopygus e Nyssomyia apresentaram ao nivel de espécies as maiores freqüências, $28,6 \%$ e $21,4 \%$ respectivamente. Mas espécies dos subgêneros Lutzomyia, 
Psathyromyia e grupo verrucarum estiveram presentes em nossas coletas.

Quanto ao número de indivíduos, as espécies Lutzomyia richardwardi $\mathrm{e}$ L. carrerai apresentaram as maiores freqüências, $39,7 \%$ e $36,2 \%$ respectivamente. Adicionamos que $L$. flaviscutellata e L. evansi (grupo verrucarum) foram coletadas somente em San Pedro (Tab. 1).

$\mathrm{Na}$ localidade de 25 de Diciembre, o predomínio do subgênero Psychodopygus, ao nível de espécies e indivíduos foi notório, $46,2 \%$ e $62,67 \%$ respectivamente. $L$. carrerai foi a espécie mais abundante com $41,9 \%$ do total de fêmeas coletadas nesta localidade, seguida de L. richardwardi $(34,1 \%)$. Ressaltamos que $L$. evangelistai e L. (Nyssomyia) olmeca nociva foram coletadas somente nesta localidade (Tab.1)

Assim também a similaridade quanto a composição dos flebotomíneos entre uma localidade e outra, segundo o indice de similaridade de Jaccard, mostra que a localidade de 25 de Diciembre foi mais similar a San Pedro e San Juan $(0,50)$, mas entre San Pedro e San Juan a similaridade foi menor, 0,47 .

Quanto às técnicas de coleta, com ambas as armadilhas foram obtidas o mesmo número de espécies (12 spp.); no entanto o maior número de indivíduos foi coletado com isca humana (61\%) (Tab. 1), assim como uma maior seletividade para as espécies antropófilas.

\section{Coletas com armadilha tipo Shannon}

Foi coletado um total de 409 flebotomíneos, sendo 95,31\% constituido por fêmeas (Tab. 1). As espécies coletadas foram agrupadas nos seguintes subgêneros: Nyssomyia (57,28\%), Psychodopygus (41,24\%), Lutzomyia $(0,74 \%)$ Psathyromyia $(0,49 \%)$ e grupo verrucarum $(0,25 \%)$.

As espécies mais abundantes capturadas com esta estratégia de coleta foram: $L$. richardwardi $(59,32 \%)$ e $L$. carrerai $(28,50 \%)$, o restante da fauna representou $12,18 \%$ do total de fềmeas coletadas (Tab. 1). Notamos que do total de machos coletados com armadilha tipo Shannon no presente estudo, $61,2 \%$ foi de espécies dos subgêneros Psychodopygus (3 spp.) e Nyssomyia (1 sp.). A maior freqüência de indivíduos (89,47\% do total de machos coletados com armadilha Shannon) é de espécies do primeiro subgênero acima mencionado. No entanto, a proporção de fêmeas para machos foi de 20:1.

L. flaviscutellata, L. olmeca nociva e $L$. evansi foram capturadas somente com armadilha tipo Shannon. Destacamos a presença das duas primeiras espécies, apesar do pequeno número de indivíduos coletados e a presença apenas de machos no caso de L. o. nociva, por serem vetores potenciais da Leishmania (Le.) amazonensis em outros locais da Amazônia (Shaw \& Lainson 1968; Lainson, 1983).

Salientamos que $L$. evansi e $L$. olmeca nociva estão sendo citadas pela primeira vez para a Amazônia Peruana.

\section{Coletas com Isca Humana}

Um total de 621 individuos, 
sendo $98,07 \%$ constituído por fềmeas, caracterizou a população de flebotomíneos coletados com esta estratégia. Lutzomyia richardwardi $(43,28 \%)$, L. carrerai $(29,18 \%), L$. hirsuta $(10,82 \%)$ e $L$. amazonensis $(9,34 \%)$ foram as espécies com os maiores números de indivíduos coletados. Todas estas espécies foram numericamente superiores as suas co - específicas coletadas com armadilha Shannon (Tab. 1). Com esta técnica de coleta a proporção de fêmeas para machos foi de 50:1.

Lutzomyia evangelistai e $L$. (Nyssomyia) yuilli pajoti foram coletadas somente com esta estratégia, embora com um escasso número de indivíduos.

De acordo com os subgêneros, em ordem de abundância, Psychodopygus com 54,50\%, Nyssomyia $43,41 \%$, Lutzomyia $1,93 \%$ e Psathyromyia $0,16 \%$ formaram a fauna de flebotomíneos.

\section{DISCUSSÃO}

O reporte de freqüentes casos de leishmaniose e a sua associação com espécies de flebotomíneos na Amazônia Peruana é pouco conhecido, assim como os vetores e reservatórios em áreas de transmissão.

O predomínio das espécies do

Tabela 1 . Número, espécie, localidade, técnica de coleta e sexo dos flebotomíneos capturados no município de Yurimaguas, Loreto, Peru.

\begin{tabular}{lcccccccccccc}
\hline \multicolumn{1}{c}{ ESPECIES } & \multicolumn{3}{c}{25 Diciembre } & \multicolumn{3}{c}{ San Pedro } & \multicolumn{7}{c}{ San Juan } \\
& \multicolumn{1}{c}{ Shannon } & I. Humana & Shannon & I. Humana & \multicolumn{2}{c}{ Shannon } & I. Humana \\
& M & F & M & F & M & F & M & F & M & F & M & F \\
\hline L. evangelistai & 0 & 0 & 0 & 2 & 0 & 0 & 0 & 0 & 0 & 0 & 0 & 0 \\
L. sherlocki & 0 & 2 & 0 & 1 & 0 & 2 & 0 & 8 & 0 & 0 & 0 & 0 \\
L. amazonensiș & 0 & 6 & 0 & 2 & 0 & 3 & 0 & 12 & 0 & 3 & 0 & 43 \\
L. carrerai carrerai & 5 & 44 & 4 & 49 & 0 & 15 & 1 & 66 & 2 & 53 & 1 & 61 \\
L. davisi & 0 & 0 & 0 & 2 & 4 & 11 & 0 & 9 & 0 & 7 & 1 & 1 \\
L. geniculata & 0 & 0 & 0 & 6 & 0 & 0 & 0 & 0 & 0 & 3 & 0 & 6 \\
L. hirsuta hirsuta & 1 & 1 & 1 & 21 & 0 & 0 & 1 & 4 & 2 & 5 & 2 & 41 \\
L. nocticola & 0 & 2 & 0 & 6 & 0 & 0 & 0 & 0 & 0 & 0 & 0 & 0 \\
L. oimeca nociva & 2 & 0 & 0 & 0 & 0 & 0 & 0 & 0 & 0 & 0 & 0 & 0 \\
L. flaviscutellata & 0 & 0 & 0 & 0 & 0 & 1 & 0 & 0 & 0 & 0 & 0 & 0 \\
L. richardwardi & 0 & 38 & 0 & 38 & 0 & 37 & 0 & 50 & 3 & 154 & 1 & 177 \\
L. yuilli pajoti & 0 & 0 & 0 & 0 & 0 & 0 & 0 & 2 & 0 & 0 & 0 & 0 \\
L. shannoni & 0 & 1 & 0 & 1 & 0 & 2 & 0 & 0 & 0 & 0 & 0 & 0 \\
L. evansi & 0 & 0 & 0 & 0 & 0 & 1 & 0 & 0 & 0 & 0 & 0 & 0 \\
TOTAL & 8 & 94 & 5 & 128 & 4 & 72 & 2 & 151 & 7 & 225 & 5 & 329 \\
\hline
\end{tabular}

I: Isca; M: Machos; F: Fêmeas 
subgênero Psychodopygus (46,7\%) em nossas coletas, é semelhante aos resultados encontrados por vários pesquisadores em áreas de floresta primária na Amazônia Brasileira (Lainson et al., 1973; Fraiha et al., 1978; Arias \& Freitas, 1982; Ready et al., 1986), assim como a sua tendência a predominar em coletas com isca humana. No entanto, Ready et al. (1986) relataram que espécies do subgênero Psychodopygus predominaram ao nivel do chão, enquanto que espécies de outro subgênero no dossel florestal (os autores referem-se a Nyssomyia) em coletas realizadas em áreas de floresta primária no Estado do Pará, deverão ser consideradas ao planejar estudos que tratem sobre o comportamento de vetores de leishmaniose do subgênero Viannia em áreas de transmissão.

Analisando os nossos resultados ao nível de indivíduos, encontramos que estes diferem dos obtidos por Fraiha et al. (1978), Arias \& Freitas (1982) e Ward et al. (1973) em diversas áreas dos Estados do Amazonas e Pará, onde o predomínio de indivíduos de espécies do subgênero Psychodopygus é nítido. É provável que as diferenças encontradas nas áreas por nós trabalhadas sejam devido à estação do ano, número de coletas e paisagem ecológica diferente daquelas dos autores acima mencionados.

Ready e colaboradores observaram que em coletas de isca humana durante a estação seca, o número de indivíduos de algumas espécies do subgênero Nyssomyia (L. umbratilis e L. yuilli pajoti) aumentava após as chuvas de verão. No entanto, Fraiha et al. (1978) e Arias \& Freitas (1982) reportaram uma diminuição na fauna de flebotomíneos deste subgênero durante a estação de menor precipitação, em áreas ecologicamente similares, utilizando as mesmas técnicas de coleta. Porém, os autores não mencionam se algumas das coletas por eles realizadas foram após ligeiras chuvas durante esta estação. A outra provável explicação para estes resultados diferentes é a paisagem ecológica ou as características ecológicas do local, fatores que segundo Forattini (1973) influenciam no comportamento e distribuição dos flebotomíneos de um local a outro.

Nós acreditamos que coletas padronizadas por um periodo de tempo mais prolongado e outras técnicas além das utilizadas na presente pesquisa que permitam estudar a distribuição vertical e longitudinal das populações de flebotomineos nas áreas estudadas, proporcionarão resultados mais conclusivos sobre o encontrado nessa pesquisa.

\section{Lutzomyia}

(Nyssomyia) richardwardi Ready \& Fraiha, a espécie mais abundante $(48,4 \%)$ em 2 das 3 localidades onde a coleta de flebotomíneos realizou-se, foi assinalada como antropófila por Ready \& Fraiha (1981) no Estado do Pará. Em outras áreas da Amazônia Brasileira foi coletada apenas com armadilhas luminosas CDC e em número reduzido (Arias \& Freitas, 1982; Biancardi et al., 1982; Castellón et al., 1994). As exceções foram os estudo de Barrett et al. (1996) no 
município de Tefé - Amazonas, onde esta espécie ocupou o terceiro lugar em ordem de abundância, e de Ready et al. (1986) no municipio de Jari - Pará, onde esta espécie predominou em floresta úmida. De acordo com o nosso conhecimento este é o primeiro reporte de coletas com isca humana desta espécie na qual domina a fauna de flebotomineos, confirmando a sua antropofilia observada por outros pesquisadores (Ready \& Fraiha, 1981).

Lutzomyia evangelistai Martins \& Fraiha e L. sherlocki Martins, Silva \& Falcão, coletada em pouco número neste estudo, foram assinaladas como espécies de provável comportamento antropófilo por Ryan et al. (1986), Castellón et al. (1994) e Barrett et al. (1996) em áreas de floresta primária nos estados do Pará e Amazonas no Brasil, respectivamente. Os nossos resultados, devido ao escasso número de indivíduos coletados, constituem apenas um aporte para ampliar o conhecimento da distribuição geográfica destas espécies na Amazônia.

Lutzomyia flaviscutellata Mangabeira coletada com armadilha tipo Shannon, confirma o citado por vários pesquisadores sobre a sua antropofilia quase nula em áreas de floresta primária na Amazônia Brasileira (Shaw \& Lainson, 1968; Forattini, 1973; Lainson et al., 1986; Ryan et al., 1986), porém sua presença é destacada pela sua importância epidemiológica na transmissão da Leishmania (Le.) amazonensis ao homem.

Lutzomyia o. nociva, L. yuilli pajoti e L.. shannoni Dyar foram reportadas como espécies de hábito alimentar variável, segundo o local (antropófilos e zoófilos), em diversas áreas florestais da Amazônia (Ward et al., 1973; Arias \& Freitas, 1977; Fraiha et al., 1978; Castellón et al., 1994). No presente estudo, o caráter antropófilo destas espécies não ficou esclarecido, devido ao número reduzido e captura apenas de machos de $L . o$, nociva (Tab 1). Acreditamos que estudos por um maior período de tempo, com técnicas de coleta diferentes e análise do sangue ingerido pelas fềmeas, ajudarão na elucidação do hábito alimentar destas espécies.

\section{AGRADECIMENTOS}

Ao Projeto de Investigações epidemiológicas de leishmanioses no departamento de Loreto, Peru, pelo financiamento desta pesquisa. Ao biólogo Máximo Viena del Aguila (in memorian) pela grande ajuda nas coletas entomológicas. Ao Dr. José Enrique Peréz Ramos e a Bióloga Elena Ogosuku, pelo auxilio na identificação do material entomológico.

\section{Bibliografias citadas}

Arias, J.R.; Freitas, R.A. 1977. Flebótomos da Amazônia Central do Brasil I. Resultados obtidos das capturas feitas com isca humana e eqüina (Diptera : Psychodidae), Acta Amazonica, 7: 507-527.

Arias, J.R.; Freitas, R.A. 1982. On the vectors of cutaneous leishmaniasis in the central Amazon of Brazil. 3. Phlebotomine sand fly stratification in a terra firme. Acta Amazonica, 12: 599-608. 
Asesoría Nacional de Planificación, 1959. Atlas histórico geográfico $y$ de paisajes peruanos, Lima, Perú. 73 p.

Blancas, F. 1959-1960. Notas sobre flebotomos peruanos. Enumeración de las especies de flebotomos encontrados en el Perú y descripción de una especie nueva: Phlebotomus gorbitzi n.sp. (Diptera : Psychodidae). Rev.Med.Exp., 12: 125-132.

Barrett, T.V.; Freitas, R.A.; Albuquerque, M.I.C.; Guerrero, J.C.H. 1996, Report on a collection of Lutzomyia sandflies (Diptera: Psychodidae) from the middle Solimões (Amazonas, Brazil). Mem.Inst.Oswaldo Cruz, 91 (1): 27-35.

Biancardi, C.B.; Arias, J.R.; Freitas, R.A.; Castellón, E.G. 1982. The known geographical distribution of sandflies in the state of Rondônia, Brazil. Acta Amazonica, 12 (1): 167-179.

Braga, J. 1987. Caracterización de Leishmanias spp. de Loreto por Patrones Iso enzimáticos. Tesis para optar el grado de Maestro en Ciencias con Mención en Bioquimica. Universidad Peruana Cayetano Heredia. 84 p.

Cáceres, A.; Galati, E.B.; Le Pont, F.; Velásquez, C. 1995. La fauna flebotomica (Diptera: Psychodidae) de tres provincias de la región nor oriental del Marañon, Perú. Rev.Soc.Bras.Med.Trop., 28 (3): 215-221.

Castellón, E.G.; Arias, J.R.; Freitas, R.A.; Naiff, R.D. 1994. Os flebotomíneos da região amazônica, estrada Manaus Humaitá, Estado do Amazonas, Brasil (Diptera : Psychodidae : Phlebotominae). Acta Amazonica, 24 (12): 91-102.

De Aguiar,M.; Soucasaux,T. 1984. Aspectos da ecologia dos flebótomos do Parque Nacional da Serra dos Órgãos, Rio de Janeiro, I. Freqüência mensal em isca humana (Díptera, Psychodidae, Phlebotominae). Mem.Inst. Oswaldo Cruz., 79(2): 197-209.

Feliciangeli, D. 1987. Ecology of sandflies (Diptera : Psychodidae) in a restricted focus of cutaneous leishmaniasis in northern
Venezuela, I. Description of the study area, catching methods and species composition. Mem.Inst. Oswaldo Cruz, 82(1): 119-124.

Forattini, O, 1973. Entomologia Médica IV. Psychodidae, Phlebotominae, Leishmaniose, Bartonellose. Edgar Blucher, São Paulo. 658 $\mathrm{p}$

Fernandez, R.; Galati, E.B.; Carbajal, F.; Wooster, M.T; Watts, D.M. 1998. Notes on the phlebotomine sandflies from the peruvian southeast - Description of Lutzomyia (Helcocyrtomyia) adamsi n. sp. (Diptera : Psychodidae). Mem.Inst. Oswaldo Cruz, 93: 41-44.

Fraiha, H.; Ward, R.; Shaw, J.; Lainson, R. 1978. Fauna antropófila de flebótomos da rodovia Transamazónica, Brasil (Diptera : Psychodidae), Bol.Ofic.Sanit. Pan., 84(2): 134-139.

Freitas, R.; Barrett, T.; Naiff, R. 1989. Lutzomyia reducta Feliciangeli et al. 1988, a host of Leishmania amazonensis, sympatric with two other members Flavicutellata complex in Southern Amazonian and Rondonia, Brazil (Diptera : Psychodidae). Mem.Inst. Oswaldo Cruz, 84(3): 363-369.

Hashigushi, Y.; Goméz, E.; Coronel, V. de; Mimori, T.; Kawabata, M. 1985. Biting activity of two anthropophilic species of sandflies, Lutzomyia, in an endemic area of leishmaniasis in Ecuador. Ann. Trop. Med. Par, 79(5): 533-537.

Herrer, A. 1977. Reseña de la Entomología Médica en el Perú, I. Principales aspectos entomológicos en la Bartonellosis, la Trypanosomiasis y la Leishmaniasis. Rev.Per.Ent., 20(1): 19-25.

Herrer, A. 1982. Lutzomyia peruensis Shannon, 1929 posible vector natural de la Uta (leishmaniasis tegumentaria). Rev. Inst. Med. Trop. S. Paulo, 24(3): 168-172.

Lainson, R. 1981. Epidemiologia e Ecologia da leishmaniose tegumentar na Amazônia. Hil.Méd., 3(1): 35-40.

Lainson, R. 1983. American Leishmaniasis: some observations their Ecology and Epidemiology. Trans.R.Soc.Trop.Med.Hyg., 77(5): 569-596. 
Lainson, R, 1988. Ecological interactions in th transmission of the leishmaniases. Phil.Trans.R.Soc.Lond. B, 321: 389-404.

Lainson, R.; Shaw, J.J. 1968. Leishmaniasis in Brazil: I. Observations of enzootic rodent leishmaniasis incrimination of Lutzomyia flaviscutellata (Mangabeira) as the vector in the lower Amazonian basin. Trans.R.Soc.Trop.Med.Hyg., 62(3): 385395.

Lainson, R.; Shaw, J.J.; Ward, R. 1973, Leishmaniasis in Brazil IX. Considerations on the Leishmania braziliensis complex: Importance of sandflies of the genus Psychodopygus (Mangabeira) in the transmission of the Leishmania b. braziliensis in North Brazil. Trans. R. Soc. Trop. Med, Hyg., 69: 165-168.

Lainson, R.; Shaw, J.; Silveira, F; Braga, R.; Ryan, L.; Póvoa, M.; Ishikawa, E. 1986. A leishmania e as leishmanioses. In: Instituto Evandro Chagas 50 anos de contribuição às ciências biológicas e à medicina tropical. Belém. Fundação Serviços de Saúde Pública. Vol. 1: 83-124

Llanos, B. 1964. Flebotomos de la Selva Peruana con la descripción de tres especies nuevas (Diptera: Psychodidae). Rev. Bras. Biol., 24(4): 371-382.

Llanos, B. 1973. Flebotomos de la Selva Peruana (Diptera: Psychodidae). Rev. Per: Ent., 16(1): 29-49.

Llanos, B. 1986. Flebotomos del Perú y su distribución geográfica. Rev.Per. Ent., 24(1): 183-184.

Llanos, B.; Martins, A.V.; Da Silva, E. 1975. Estudos sobre os flebotomíneos do Peru (Díptera, Psychodidae, Phlebotominae) I. Departamento do Cuzco 3.- Descrição do macho e redescrição da fêmea de Lutzomyia (Psychodopygus) amazonensis (Root, 1934) e lista das especies coletadas. Rev. Bras. Biol., 35: 665-672.

Ludwig, AJ.; Reynolds, J.F. 1988. Statistical Ecology. - A primer on methods and computing. A Wiley Interscience Publication New York. 337 p,

Martins, A.V.; Fraiha,H. 1971. Lutzomyia evangelistai nova espécie de flebótomo do Estado do Pará (Diptera, Psychodidae,
Phlebotominae). Rev.Bras.Biol., 31(3): 361-365.

Maroli, M.; Feliciangeli, D.; Arias, J.R. 1995. Métodos de Captura Consevación y Montaje de los Flebotomos (Díptera : Psychodidae). O.P.S e O.M.S. (publicación no oficial) ISBN 927532232 5., 72 p.

ONERN. 1976. Mapa Ecológico del Perú. Guia Explicativa Lima. 146 p.

Peréz, E.; Villaseca, P.; Llanos-Cuentas, A.; Guerra, H. 1987. Técnicas para colectar titiras (Lutzomyia spp. Diptera :Psychodidae) en ambientes altoandinos peruanos. Rev.Per.Ent., 30: 77-80.

Ready, R.; Fraiha, H. 1981. Brazilian Phlebotomines VI. Lutzomyia richardwardi sp.n, a new species of Nyssomyia from Amazonian with keys for the subgenus (Diptera:Psychodidae). Rev. Bras. Biol., 41(4): 705-712.

Ready, P.; Lainson, R.; Shaw, J.; Ward, R. 1986. The ecology of Lutzomyia umbratilis Ward \& Fraiha (Diptera : Psychodidae) the major vector to man of Leishmania braziliensis guyanensis in north-eastern Amazonian Brazil. Bull.Ent.Res., $\quad$ 76: 21-40.

Ryan, L.; Lainson, R.; Shaw, J.; Fraiha, H. 1986. Ecologia de flebotomíneos (Díptera : Psychodidae) na região amazonica. In: Instituto Evandro Chagas 50 anos de contribuição às ciências biológicas e à medicina tropical. Belém, Fundaçào Serviços de Saúde Pública. Vol. 1: 307-320.

Ryan, L.; Lainson, R.; Shaw, J.; Braga, R.; Ishikawa, E. 1987. Leishmaniasis in Brazil. XXV. Sandfly vectors of Leishmania in Pará State, Brazil. Med.Vet Ent., 1: 383-395.

Shaw, J.J.; Lainson, R. 1968. Leishmaniasis in Brazil II. Observations on enzootic rodent leishmaniasis in the lower amazon regionThe feeding habits of the vector, Lutzomyia flaviscutellata in reference to man, rodents and other animals. Trans. R.Soc.Trop.Med.Hyg., 62(3): 396-405.

Villarejo, A. 1979. Asi es la Selva. 3a edic., Edit. CETA, Iquitos, Perú. $231 \mathrm{p}$. 
Villavicencio, M.F. 1985. Memoria AnualInstituto Nacional de Investigaciones y Promoción Agropecuaria XVI. Estación Experimental de Yurimaguas. $14 \mathrm{p}$.

Ward, R.; Shaw, J.; Lainson, R.; Fraiha, H. 1973. Leishmaniasis in Brazil: VIII. Observations on the Phlebotomine fauna of an area highly endemic for cutaneous leishmaniasis, in the Serra dos Carajás, Pará State. Trans.R. Soc.Trop.Med.Hyg., 67(2): 174-183.

Young, D. 1979. A review of the bloodsucking psychodid flies of Colombia (Diptera: Phlebotominae and Sycoracinae) Tech. Bull. 806, Agr.Exp.Stn., IFA, Univ. Florida. 226 p.

Young, D.; Peréz, E.; Romero, G. 1985. New records of Phlebotomine sandflies from Peru with a description of Lutzomyia oligodonta n.sp. from the Rimac Valley (Diptera:Psychodidae). Int.J.Ent., 27(1): 136-146. 